\title{
Systemic steroids in patients with COVID-19: pros and contras, an endocrinological point of view
}

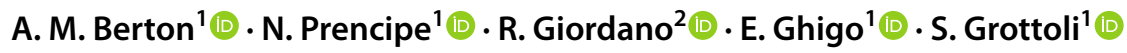

Received: 22 May 2020 / Accepted: 2 June 2020 / Published online: 8 June 2020

(C) Italian Society of Endocrinology (SIE) 2020

Keywords Coronavirus · Glucocorticoids · Adrenal insufficiency · Viral pneumonia · ARDS · Septic shock

Systemic corticosteroids are not currently recommended for the treatment of lung injury related to the Severe Acute Respiratory Syndrome-CoronaVirus-2 (SARS-CoV-2) infection [1]. In the past, steroid administration did not clearly improve the mortality rate of patients affected by SARSCoV-related pneumonia against possible collateral effects and seemed to reduce the clearance in the respiratory tract of Coronavirus associated with Middle Eastern Respiratory Syndrome (MERS-CoV) [1]. Nevertheless, corticosteroids are often used in viral pneumonia, particularly when specific conditions and comorbidities are present (asthma exacerbation, chronic obstructive pulmonary disease, septic shock refractory to fluid resuscitation and vasopressor administration).

During the current pandemic of 2019 Coronavirus Disease (COVID-19), many therapeutic protocols adopted in Intensive Care Units (ICUs) and Infectious Disease Departments include high dose systemic corticosteroids for the treatment of moderate to severe respiratory insufficiency [2], as indicated in the Acute Respiratory Distress Syndrome (ARDS). Even in ARDS non-related to COVID-19, the main indications for steroid treatment are the probable response of the underlying pathological triggering process and patient's comorbidities; in the absence of these conditions, there is only a modest evidence of reduced mortality when corticosteroids are administered early (within the first 14 days) in the presence of persistent severe respiratory failure despite of standard therapy [3].

S. Grottoli

silvia.grottoli@unito.it

1 Division of Endocrinology, Diabetology and Metabolism, Department of Medical Sciences, University of Turin, Corso Dogliotti 14, 10126 Turin, Italy

2 Department of Biological and Clinical Sciences, University of Turin, Turin, Italy
As endocrinologists, we do believe the potent anti-inflammatory properties of synthetic steroids are clear-cut [4] and, to our knowledge, definitive evidence that other anti-inflammatory strategies provide better efficacy is still lacking, at least in COVID-19.

The specific mechanism by which steroids would act on sustained lung inflammation, as well as the definition of the best drug to use and even the adequate treatment duration are still objectives of ongoing clinical trials.

Indeed, although synthetic steroids share a marked antiinflammatory action and poor mineralocorticoid effects, their bioequivalence and different kinetics must be carefully considered. Hydrocortisone is indicated in septic shock at a dose of 200-400 mg/day to avoid Critical Illness Related Corticosteroid Insufficiency (CIRCI); it presents a little antiinflammatory action and should be administered every $8 \mathrm{~h}$ due to its reduced half-life. Methylprednisolone, instead, can be indicated in severe ARDS at a dose of $0.5-2 \mathrm{mg} / \mathrm{kg} / \mathrm{day}$; it shows a prolonged half-life together with a good penetration into lung tissue and can be administered once-a-day. Finally, dexamethasone, the most powerful synthetic steroid, widely used for its marked anti-edema properties, provides the most consistent duration of action and, recently, a large randomized controlled trial suggested its efficacy in moderate to severe ARDS patients [5].

The use of similar doses of potent corticosteroids requires a profound knowledge of the related side effects (e.g. avascular necrosis, psychosis, diabetes), their adequate prevention and a prompt treatment if necessary.

The ability of synthetic steroids to induce a persistent inhibition of the Hypothalamic-Pituitary-Adrenal (HPA) axis, even after a short period of treatment, is not clearly predictable due to the high inter-individual variability in pharmacokinetics and in Glucocorticoid-Receptors (GRs) sensitivity. Moreover, the pathological changes in cortisol metabolism and action in critically ill patients (reduced 
cortisol degradation, altered binding protein levels, etc.) are well known.

On this basis, parenteral administration of high doses of methylprednisolone (e.g. up to $8 \mathrm{mg} / \mathrm{Kg} /$ day for variable time in our Country) or dexamethasone (e.g. $20 \mathrm{mg} /$ day for 5 days, then $10 \mathrm{mg} /$ day for 5 days), besides monitoring of side effects, requires a cautious dose tapering toward substitutive dose likely to be continued until the end of the acute phase of COVID-19.

Whenever the withdrawal of synthetic steroids is done, careful attention should be given to a consequent phase of adrenal insufficiency $[6,7]$. Considering that the evaluation of cortisol concentrations and its response to ACTH stimulation are unlikely feasible in critically ill patients, the opportunity of a transient replacement treatment with hydrocortisone should be considered until the end of the disease. This approach is supported by other relevant aspects.

The occurrence of adrenal insufficiency is even more likely in therapeutic schemes including ritonavir (RTV), an antiviral drug which is also a powerful inhibitor of the cytochrome P450 3A4 (CYP3A4). By increasing the concentrations of the other drugs metabolised by the same pathway, such as corticosteroids, this protease inhibitor is able to promote a iatrogenic hypercortisolemic condition, which in turn leads to a reduced synthesis and release of CRH and ACTH, inducing a subsequent hypocortisolemic state [8]. Therefore, the abrupt discontinuation of a systemic steroid, particularly when associated with RTV, could be hazardous in ICUs patients being able to hesitate in open adrenal insufficiency. At the same time, the frequent side effects of other combination treatments, such as hydroxychloroquine (i.e. hypoglycaemia) and antiviral drugs (i.e. diarrhea), could mask a new onset hypocortisolemic state, which conversely would promptly require adrenal replacement therapy.

Moreover, due to the high homology with the structure of the original SARS-CoV, also antibodies produced against SARS-CoV-2 have been hypothesized to cross-react with the ACTH peptide, potentially contributing to a relative cortisol deficiency [9].

Even more important, adrenal insufficiency is associated to a significant reduction in the natural killer cell function that would in turn impair the recognition and elimination of virally infected cells [10]. Consequently, hypocortisolemic patients should have to be monitored for a possible resurgence of the infectious state, but, despite this, the novel acquired antiviral immune deficiency could be associated with an increased mortality in respiratory infections such as COVID-19.

In all, although the administration of supraphysiological doses of corticosteroids in viral pneumonia is still matter of debate [2-5], based on the previous considerations, we believe that systemic steroids should not be precluded to critically ill patients, providing the correct dose scaling.
We should consider that the normal adrenal response to stress is estimated at a five- to ten-fold increase, and there is no doubt that COVID-19 infection represents a condition of dramatic and prolonged stress; on the other hand, we don't know almost anything about the capability of the endogenous HPA axis to appropriately respond in this condition. As an adequate HPA response to COVID-19 infection is needed, but we cannot rely on the evidence that this takes place, we believe that this should represent one more good reason to consider corticosteroids for treatment of these patients.

Author contributions All authors contributed to the conception of the manuscript. All authors reviewed the text and approved the final version.

Funding This manuscript did not receive any specific grant from any funding agency or pharmaceutical company.

\section{Compliance with ethical standards}

Conflict of interest On behalf of all authors, the corresponding author states that there is no conflict of interest.

Ethical approval This article does not contain any studies with human participants or animals performed by any of the authors.

Informed consent For this type of study, formal consent is not required.

\section{References}

1. WHO (2020) Clinical management of severe acute respiratory infection when COVID-19 is suspected. https://www.who.int/ publications-detail/clinical-management-of-severe-acute-respi ratory-infection-when-novel-coronavirus-(ncov)-infection-issuspected. Accessed 21 May 2020.

2. Shang L, Zhao J, Hu Y et al (2020) On the use of corticosteroids for 2019-nCoV pneumonia. Lancet 395:683-684. https://doi. org/10.1016/S0140-6736(20)30361-5

3. Lewis SR, Pritchard MW, Thomas CM, Smith AF (2019) Pharmacological agents for adults with acute respiratory distress syndrome. Cochrane Database Syst Rev. https://doi. org/10.1002/14651858.CD004477.pub3

4. Isidori AM, Arnaldi G, Boscaro M et al (2020) COVID-19 infection and glucocorticoids: update from the Italian Society of Endocrinology Expert Opinion on steroid replacement in adrenal insufficiency. J Endocrinol Invest. https://doi.org/10.1007/s4061 8-020-01266-w

5. Villar J, Ferrando C, Martínez D et al (2020) Dexamethasone treatment for the acute respiratory distress syndrome: a multicentre, randomised controlled trial. Lancet Respir Med 8:267-276. https://doi.org/10.1016/S2213-2600(19)30417-5

6. Scaroni C, Armigliato M, Cannavò S (2020) COVID-19 outbreak and steroids administration: are patients treated for Sars-Cov-2 at risk of adrenal insufficiency? J Endocrinol Invest. https://doi. org/10.1007/s40618-020-01253-1

7. Annane D, Pastores SM, Rochwerg B et al (2017) Guidelines for the Diagnosis and Management of Critical Illness-Related 
Corticosteroid Insufficiency (CIRCI) in Critically Ill Patients (Part I): Society of Critical Care Medicine (SCCM) and European Society of Intensive Care Medicine (ESICM) 2017. Crit Care Med 45:2078-2088. https://doi.org/10.1097/CCM.0000000000002737

8. Wood BR, Lacy JM, Johnston C et al (2015) Adrenal insufficiency as a result of ritonavir and exogenous steroid exposure: report of 6 cases and recommendation for management. J Int Assoc Provid AIDS Care 14:300-305. https://doi.org/10.1177/2325957414 567681

9. Wheatland R (2004) Molecular mimicry of ACTH in SARS: implications for corticosteroid treatment and prophylaxis. Med Hypotheses 63:855-862. https://doi.org/10.1016/j. mehy.2004.04.009
10. Bancos I, Hazeldine J, Chortis V et al (2017) Primary adrenal insufficiency is associated with impaired natural killer cell function: a potential link to increased mortality. Eur J Endocrinol 176:471-480. https://doi.org/10.1530/EJE-16-0969

Publisher's Note Springer Nature remains neutral with regard to jurisdictional claims in published maps and institutional affiliations. 